\title{
A novel membrane-dependent on/off switch mechanism of talin FERM domain at sites of cell adhesion
}

\author{
Xianqiang Song ${ }^{1,2,{ }^{*}}$, Jun Yang ${ }^{2, *}$, Jamila Hirbawi ${ }^{2}$, Sheng Ye ${ }^{1}, \mathrm{H}_{\text {Dhanuja Perera }}{ }^{2}$, Esen Goksoy ${ }^{2}$, \\ Pallavi Dwivedi', Edward F Plow ${ }^{2}$, Rongguang Zhang ${ }^{1}$, Jun Qin ${ }^{2}$ \\ ${ }^{I}$ Institute of Biophysics, Chinese Academy of Sciences, Beijing, China; ${ }^{2}$ Department of Molecular Cardiology, Lerner Research In- \\ stitute, Cleveland Clinic, 9500 Euclid Ave, Cleveland, OH 44195, USA
}

The activation of heterodimeric $(\alpha / \beta)$ integrin transmembrane receptors by cytosolic protein talin is crucial for regulating diverse cell-adhesion-dependent processes, including blood coagulation, tissue remodeling, and cancer metastasis. This process is triggered by the coincident binding of N-terminal FERM (four-point-one-protein/ezrin/radixin/moesin) domain of talin (talin-FERM) to the inner membrane surface and integrin $\beta$ cytoplasmic tail, but how these binding events are spatiotemporally regulated remains obscure. Here we report the crystal structure of a dormant talin, revealing how a C-terminal talin rod segment (talin-RS) self-masks a key integrin-binding site on talinFERM via a large interface. Unexpectedly, the structure also reveals a distinct negatively charged surface on talinRS that electrostatically hinders the talin-FERM binding to the membrane. Such a dual inhibitory topology for talin is consistent with the biochemical and functional data, but differs significantly from a previous model. We show that upon enrichment with phosphotidylinositol-4,5-bisphosphate (PIP2) - a known talin activator, membrane strongly attracts a positively charged surface on talin-FERM and simultaneously repels the negatively charged surface on talinRS. Such an electrostatic "pull-push" process promotes the relief of the dual inhibition of talin-FERM, which differs from the classic "steric clash" model for conventional PIP2-induced FERM domain activation. These data therefore unravel a new type of membrane-dependent FERM domain regulation and illustrate how it mediates the talin on/off switches to regulate integrin transmembrane signaling and cell adhesion.

Keywords: cell adhesion; FERM domain; integrin signaling; crystallography; NMR

Cell Research (2012) 22:1533-1545. doi:10.1038/cr.2012.97; published online 19 June 2012

\section{Introduction}

Talin was discovered nearly three decades ago as a highly abundant cytosolic protein important for cytoskeleton organization and cell-extracellular matrix (ECM) adhesion [1]. Extensive genetic and cell biological studies have established that talin is crucial for regulating a wide variety of integrin-mediated cell adhesiondependent processes, such as cell-shape change, growth,

*These two authors contributed equally to this work. Correspondence: Jun Qin ${ }^{\mathrm{a}}$, Rongguang Zhang ${ }^{\mathrm{b}}$

${ }^{\mathrm{a}}$ Tel: +1-216-444-5392; Fax: +1-216-445-1466

E-mail: qinj@ecf.org

${ }^{\mathrm{b}}$ Tel: 010-64888786

E-mail: rzhang@sun5.ibp.ac.cn

Received 17 February 2012; revised 6 May 2012; accepted 15 May 2012; published online 19 June 2012 differentiation, and migration [2-4]. Talin is large in size, with 2541 amino acids, and can be divided into two major segments, an N-terminal head (1-433, talin-H, $50 \mathrm{kDa}$ ) that contains a FERM (four-point-one-protein/ ezrin/radixin/moesin) domain (86-400, talin-FERM) and a C-terminal rod (482-2541, talin-R, $220 \mathrm{kDa}$ ) that contains a series of consecutive helical bundles followed by an actin-binding motif [2-4]. Talin-FERM, which engages with heterodimeric $(\alpha / \beta)$ integrin adhesion receptors, can be further divided into F1, F2, and F3 subdomains, with F3 specifically interacting with integrin $\beta$ cytoplasmic tails (CTs) [2-4]. Because of its capacity to bind both integrin and actin, talin has long been recognized as a mechanical linker between the ECM and actin cytoskeleton to regulate cell adhesion and morphology [5]. However, about a decade ago, it was found that the binding of talin-FERM to integrin $\beta$ CTs can also promote the conversion of integrins from a low-affinity into 
a high-affinity ligand-binding state, a dynamic process termed integrin inside-out signaling or integrin activation [6-9]. This finding suggests that the talin-FERM/ integrin interaction plays a multifunctional role in promoting integrin activation as well as integrin-actin coupling during dynamic cell adhesion processes [10-12]. Structural and biochemical analyses have shown that the talin-FERM binding to integrin $\beta$ CT triggers the separation of a key integrin $\alpha / \beta C \mathrm{C}$ clasp, thus facilitating the global conformational change and activation of the receptor $[7,8,13]$. Stretches of positively charged surfaces on F1, F2, and F3 were also found to be crucial for this process by targeting talin-FERM to the membrane and enhancing the talin-FERM/integrin $\beta$ CT interaction [13-17]. However, how the membrane-targeting and integrin binding of talin-FERM are spatiotemporally regulated to control the dynamics of integrin adhesion is not understood. This issue is important, since uncontrolled talin activity may lead to dysfunction of integrins, which is linked to many human disorders, including thrombosis, stroke, bleeding, infections, and cancer metastasis [18]. Talin can adopt inactive and active states during dynamic cell adhesion events, but the underlying molecular basis for this transition is elusive [2]. Previous studies suggested that the inactive talin has an autoinhibited conformation, where a key integrin-binding site on talin-F3 is self-masked by a talin-R segment (talinRS) $[19,20]$. Membrane lipid phosphotidylinositol-4,5bisphosphate (PIP2) [19, 21], which is locally enriched by talin-recruited PIPKI $\gamma$ kinase $[22,23]$, was shown to activate talin $[19,21]$ and promote integrin-mediated cell adhesion [15, 24-28], but the detailed structural basis of this is not clear. The prevailing hypothesis is that PIP2 may sterically induce the conformational change of talin $[2,15,16,19,21]$, a mechanism widely employed in the known PIP2-mediated activation of proteins, including FERM domain proteins [29, 30].

Here we describe the first high-resolution crystal structure of the principal autoinhibitory unit of talin. The structure reveals an unexpected dual inhibitory topology in which talin-RS not only sterically masks the integrinbinding site on talin-FERM via one large interface, but also electrostatically hinders the membrane-targeting of talin-FERM via another extensively negatively charged surface. Such a topology is consistent with the biochemical and functional data, but strikingly different from a previous model [20]. The topology further suggests a "pull-push" mechanism for talin activation by membrane enriched with PIP2, which differs distinctly from the classic steric-clash mechanism for the conventional PIP2-mediated activation of FERM proteins activation. We verify this "pull-push" mechanism by a combina- tion of nuclear magnetic resonance (NMR), biochemi$\mathrm{cal}$, and functional experiments. Our data lay down a new foundation for understanding talin inhibition and activation through novel regulatory mechanisms. They also provide significant insight into how the changes of the membrane surface promote an on/off switch of a cytosolic protein to control the receptor transmembrane signaling - an emerging area in signal transduction that is poorly explored [30].

\section{Results}

\section{Crystal structure of autoinhibited talin}

Previous structural mapping experiments have shown that an interdomain complex between talin-F3 and the C-terminal rod fragment (1654-1848; talin-RS) represents the principal structural unit for talin autoinhibition [19]. Talin-R 1984-2344 fragment was also found to interact with talin-F3, but at a low affinity $(\sim 20$-fold weaker than the talin-RS/talin-F3 interaction) unrelated to talin autoinhibition [19]. To obtain a high-resolution view of the autoinhibited talin, we screened multiple constructs related to the talin-RS/talin-F3 complex for crystallization, among which talin-F2F3 (206-405) in complex with talin-RS was eluted by gel filtration and crystallized, allowing us to solve its structure (Figure $1 \mathrm{~A}$ ) at $2.0 \AA$ resolution (see structural statistics in Table 1). Talin-F2F3 and talin-F3 bind to talin-RS identically as judged by NMR, indicating that F2 does not affect the talin-F3/talin-RS interface (Supplementary information, Figure S1A). In the complex, talin-F2F3 and talinRS exhibit the same fold as their free forms [20, 31], but pack extensively with a buried interface of $1750 \AA^{2}$ where the five-helix bundle of talin-RS is held in place by two protruding fingers of talin-F3 (Figure 1A). Superposition of talin-F2F3 bound to talin-RS with that bound to the integrin $\beta 1 \mathrm{CT}$ [13] (PDB code 3G9W) shows that a major portion of membrane-proximal region but not the membrane-distal region of integrin $\beta 1 \mathrm{CT}$ is sterically blocked by the talin-F2F3/talin-RS interface (Figure 1B). The autoinhibitory interface is largely hydrophilic, but also contains hydrophobic interactions as summarized in Figure 1C. Notably, (i) the carboxyl group of E1770 of talin-RS forms a buried salt-bridge with K318$\mathrm{NH}_{3}{ }^{+}$and a strong hydrogen bond with the backbone of G321, and van der Waals contact with the backbone of M319 of talin-F3; (ii) the hydroxyl group of T1767 hydrogen bonds with $\mathrm{K} 318$ side chain $-\mathrm{NH}_{3}{ }^{+}$, N323 side chain $-\mathrm{NH}_{2}$, and backbone carbonyl of $\mathrm{K} 322$, respectively, and the methyl group of T1767 also makes van der Waals contacts with G321 and N323, respectively; (iii) the side chain of M1802 exhibits hydrophobic interac- 
Table 1 Talin-F2F3/talin-RS complex diffraction data and refinement statistics

\begin{tabular}{|c|c|}
\hline \multicolumn{2}{|l|}{ Crystallographic data } \\
\hline $\mathrm{d}_{\min }(\AA)$ & $2.05(2.05 \sim 2.10)^{\mathrm{a}}$ \\
\hline Measured reflections & 19,565 \\
\hline Average redundancy & 8.8 \\
\hline Mean I/ $\sigma(\mathrm{I})$ & 15.8 \\
\hline Completeness $(\%)$ & $87.8(51.2)$ \\
\hline$R_{\text {merge }}^{\mathrm{b}}$ & $0.150(0.519)$ \\
\hline \multicolumn{2}{|l|}{ Refinement statistics } \\
\hline Bragg spacing $(\AA)$ & $34.89-2.05$ \\
\hline Space group & $\mathrm{P} 2{ }_{1} 2_{1}$ \\
\hline \multicolumn{2}{|l|}{ Cell parameters } \\
\hline $\mathrm{a}(\AA)$ & 36.7 \\
\hline $\mathrm{b}(\AA)$ & 86.9 \\
\hline $\mathrm{c}(\AA)$ & 113.6 \\
\hline Reflections in working set & 18,519 \\
\hline Reflections in test set & 1046 \\
\hline$R_{\text {cryst }}{ }^{\mathrm{c}}$ & 0.210 \\
\hline$R_{\text {free }}{ }^{\mathrm{d}}$ & 0.249 \\
\hline r.m.s.d. bonds $(\AA)$ & 0.013 \\
\hline r.m.s.d. angles $\left({ }^{\circ}\right)$ & 1.579 \\
\hline Average $B$-factor $\left(\AA^{2}\right)$ & 51.9 \\
\hline \multicolumn{2}{|c|}{$\begin{array}{l}{ }^{c} R_{\text {cryst }}=\left(\Sigma|| F_{\mathrm{o}}|-| F_{\mathrm{c}} \mid\right) / \Sigma\left|F_{\mathrm{o}}\right| \text {, where } F_{\mathrm{o}} \text { and } F_{\mathrm{c}} \text { denote observed and cal- } \\
\text { culated structure factors, respectively. } \\
{ }^{d} R_{\text {free }} \text { is equivalent to } R_{\text {cryst }} \text {, but calculated using randomly chosen } 10 \% \\
\text { reflections as the test set, which were excluded from refinement pro- } \\
\text { cess. }\end{array}$} \\
\hline
\end{tabular}

tions with the methyl group of M319 and the hydrophobic part of K322 side chain; (iv) the carboxyl group of D1809 hydrogen bonds with the carboxamide of N323; (v) the carboxamide of Q1677 hydrogen bonds with the hydroxyl group of Y377; (vi) the carboxamide of N1669 hydrogen bonds with the carboxyl group of D375; (vii) the carboxyl group of D1676 hydrogen bonds with the backbone amide of Y377; and (viii) the side chains of V1683 and L1680 exhibit hydrophobic interactions with A360.

The autoinhibitory interface of the crystal structure is consistent with the previously reported result that the L325R mutation had no effect on the complex formation [19], since L325 is not in the complex interface (Figure 1C). It is also consistent with extensive loss-offunction mutagenesis data $[19,20]$, including mutations of M319A, S365D, S379R, Q381V, T1767A/D/E, and
E1770A, which all perturb the interface environment. Specifically, M319A, T1767A/D/E, and E1770A directly disrupt the interface based on the above described interactions. The S365D mutation reduces the talin-RS/talinF3 interaction [19], because it introduces a negatively charged group that would interact unfavorably with L1680 and V1683. Similarly, S379R and Q381V mutations introduce side chains with different properties, thereby perturbing the surrounding interface and reducing the talin-RS/talin-F3 binding [19]. The effect of the M319A mutation in relation to the structure is particularly notable, since this mutation was shown to constitutively activate talin [19]. On the basis of our structure (Figure 1C), the mutation would impair a hydrophobic network of the M319 side chain with M1802 and the hydrophobic portions of K322 and K324 side chains (Figure 1C). This network is crucial for stabilizing the K318-K324 finger conformation that accounts for $~ 50 \%$ of the autoinhibitory interactions. Indeed, the position of the K318-K324 loop is significantly shifted in the autoinhibited form as compared to the active integrin-bound form (see the arrow in Figure 1B), indicating different conformations of the finger at different states. In Supplementary information, Figure S1, we further confirmed experimentally that the M319A mutation (Supplementary information, Figure S1B) or the K322A/K324A mutation (Supplementary information, Figure S1C) significantly impaired talin-F2F3 binding to talin-RS.

The crystal structure is drastically different from the previously reported NMR-based model that was primarily built by the HADDOCK program using ambiguous chemical shift constraints [20]. Overlay of talin-F3 between the crystal structure and the HADDOCK model reveals that the bound talin-RS in the crystal structure is rotated nearly $90^{\circ}$ versus the model (Supplementary information, Figure S1D). The interface of the HADDOCK model would cause steric clash with PIPKI $\gamma$ (Supplementary information, Figure S1E, right panel), which is incompatible with the previous competition experiment showing that PIPKI $\gamma$ had little effect on the talin-F3/talin-RS complex formation [19]. By contrast, the competition data are fully consistent with the crystal structure which shows no steric clash with PIPKI $\gamma$ (Supplementary information, Figure S1E, left panel). The interface of the HADDOCK model is also inconsistent with the mutagenesis data. Notably, L325 is in the core interface of the HADDOCK model (Supplementary information, Figure S1F) [20], but its mutation to Arg had no effect on the talin-F3/talin-RS complex formation [19]. Also, M319 is not in the complex interface of the HADDOCK model and does not form any interaction network [20] (Supplementary information, Figure S1F), 

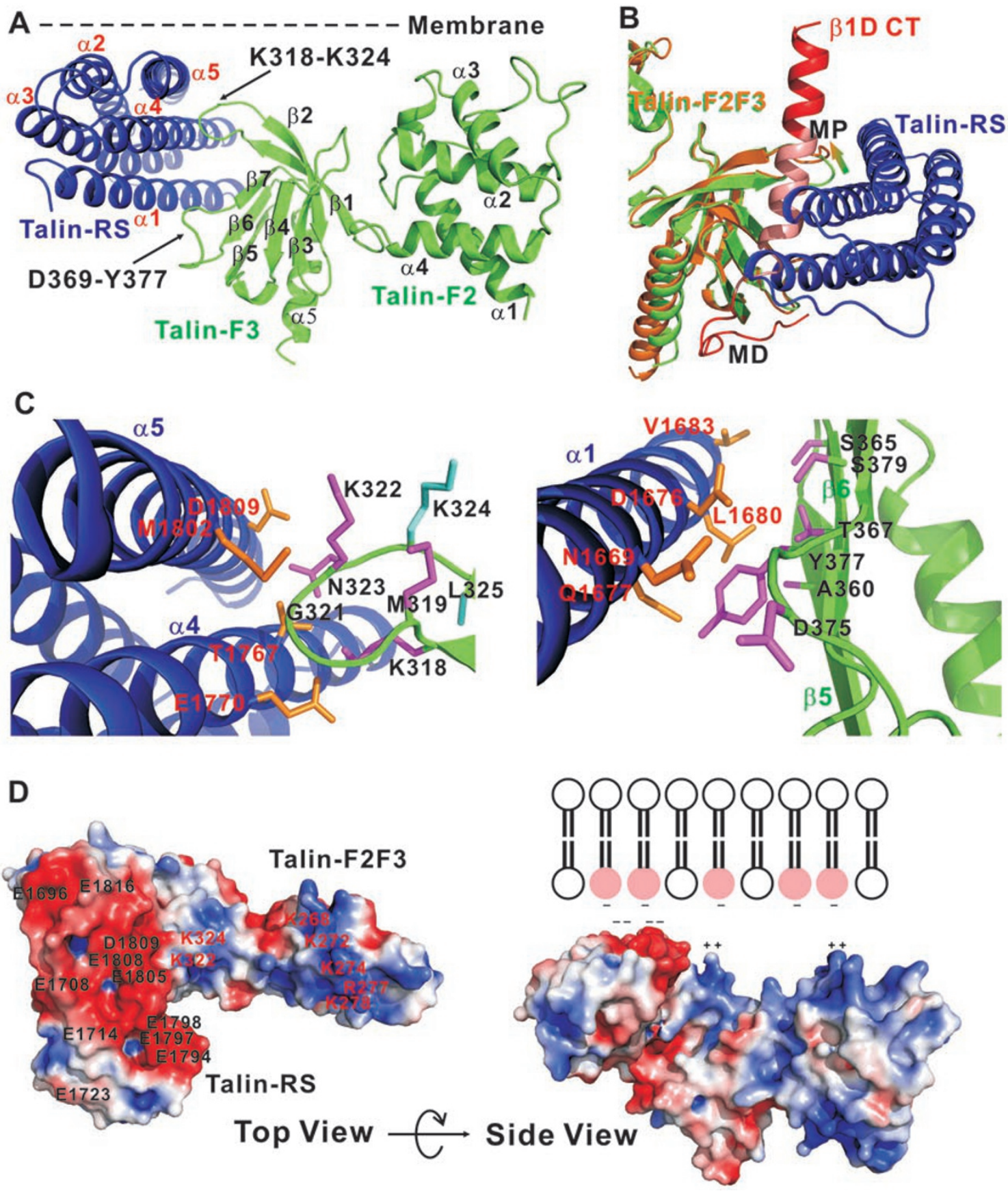

Figure 1 Talin-RS has a dual inhibitory role on talin-F2F3. (A) Crystal structure of talin-F2F3/talin-RS complex. The individual subunits are labeled in green for talin-F2F3 and blue for talin-RS. Arrows indicate the two fingers in talin-F2F3 grabbing talinRS. (B) Superimposition of talin-F2F3 bound to talin-RS with that bound to $\beta 1 D$ CT (PDB code 3G9W) showing that the membrane proximal (MP, salmon) region, but not the membrane distal (MD, red) region in $\beta 1 \mathrm{D} C T$ is buried in the talin-F2F3/talinRS interface. The arrow indicates the significant movement of K318-K324 loop in talin-F3 from the inactive state (bound to talin-RS) to active state (bound to $\beta 1 D C T$ ). (C) Close-up view of talin-F2F3/talin-RS interface shown in Figure 1A. Left panel shows the amino acid side chains involved in upper finger region (K318-K324) and right panel shows the ones in bottom finger region (D369-Y377) and the $\beta 5$ and $\beta 6$ regions. Interface residues from talin-F3 are colored in pink and labeled in black; interface residues from talin-RS are colored in orange and labeled in red; non-interface residues K324 and L325 in talin-F3 are colored in cyan. (D) Electrostatic surface representation of the complex viewed from the top (left panel) and from the side (right panel). The blue area indicates positive charge enriched region and red area indicates negative charge enriched region. It is clear that the extensively negatively charged surface on talin-RS would disfavor the talin association with membrane (right panel). 


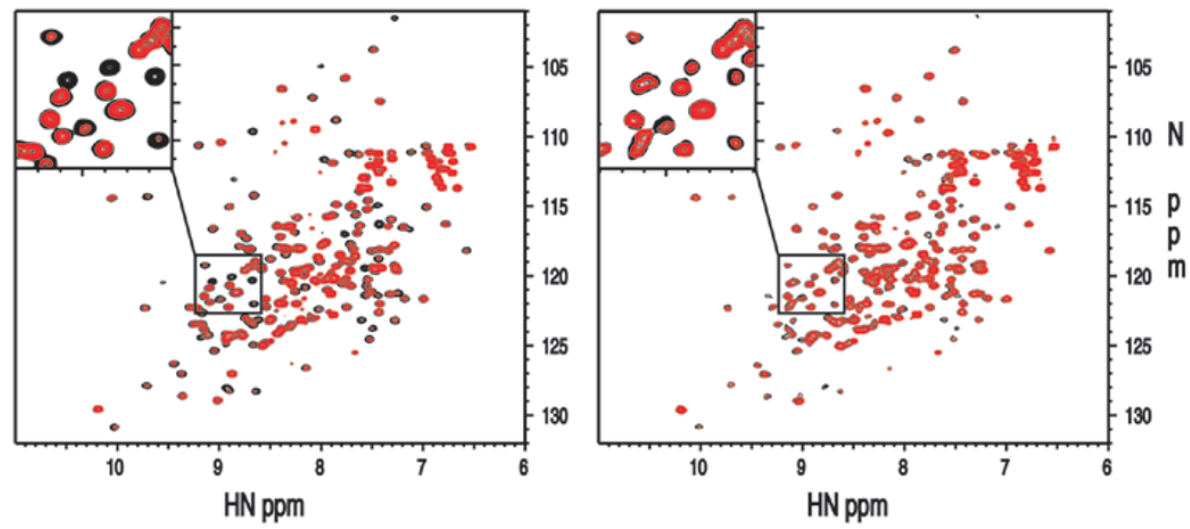

Figure 2 The autoinhibited talin is unfavorable to bind membrane. $2 \mathrm{D}-\mathrm{HSQC}$ of $0.04 \mathrm{mM}{ }^{15} \mathrm{~N}$-labeled talin-F2F3 (left) or 0.04 $\mathrm{mM}{ }^{15} \mathrm{~N}$-labeled talin-F2F3 plus $0.08 \mathrm{mM}$ unlabeled talin-RS (right) in the absence (black) and presence (red) of $2.4 \mathrm{mM} 4: 1$ POPC:POPS LUV. Significant line broadening (as reflected by the decreased peak intensity) occurred for many signals (see representative insert panel) when negatively charged vesicle was added to free talin-F2F3 (left panel). Such line broadening effect was dramatically reduced (right panel) when the same vesicles were added to talin-F2F3/talin-RS complex, indicating talin-RS effectively inhibits the access of talin-F2F3 to membrane.

which fails to explain why its mutation to Ala weakens the talin-F3/talin-RS interaction (Supplementary information, Figure S1B) and constitutively activates talin [19]. These data together indicate that the crystal structure represents a physiologically relevant form of inactive talin. The crystal structure also illustrates clearly how the inactive talin has a low capacity to bind and activate integrin (Figure 1B), and provides a template for understanding how talin may be activated (see below).

Talin-RS exhibits a negatively charged surface that electrostatically inhibits the talin-F2F3 binding to membrane

As mentioned above, talin-FERM contains a stretch of positively charged surface that associates with membrane to promote the talin-F3/ $\beta$ integrin-MPCT interaction [13-17]. Figure 1D (left panel) provides the detailed illustration of the positively charged surface in talinF2F3, which mainly involves K268, K272, K274, R277, and K278 in talin-F2, and K322 and K324 in talin-F3. This positively charged surface appears to play a dominant role in the membrane anchoring of talin-FERM [14, $15,28]$. Interestingly, careful examination of the talinF2F3/talin-RS complex structure revealed a large negatively charged surface on talin-RS, which is located at the same side as the positively charged surface of talinF2F3 (Figure 1D, left panel). This finding immediately suggested to us that the membrane binding of talin-F2F3 in the latent talin would be inhibited by the negatively charged surface of talin-RS via charge-charge repulsion (Figure 1D, right panel). This prediction was readily verified by the NMR-based competition experiments showing that while free talin-F2F3 binds to large uniamellar vesicles (LUV) containing the negatively charged lipid, 1-palmitoyl-2-oleoyl-sn-glycero-3-[phospho-Lserine] (POPS; Figure 2, left panel), the binding is significantly inhibited upon addition of talin-RS (Figure 2, right panel). Since talin-RS does not bind LUV (data not shown), the only possibility for such inhibition to occur, based on our structure, is through charge-charge repulsion between talin-RS and the membrane when talin-RS is bound to talin-F2F3 (Figure 1D, right panel). Thus, the autoinhibited talin-F2F3/talin-RS complex has a unique conformation that is sterically inhibited for binding to integrin (Figure 1B) and electrostatically hindered for binding to membrane (Figure 1D, right panel).

\section{PIP2 binds to talin-F2F3 in a distinct bivalent mode}

If the autoinhibited talin is unfavorable for membrane binding, how would talin be activated by PIP2 that binds to talin-F2F3 [15, 19, 21]? To understand this process, we first used NMR to compare the specificities of talinF2F3 binding to LUV enriched with POPS, PIP2, and POPC (1-palmitoyl-2-oleoyl-sn-glycero-3-phosphocholine), all of which are found on the inner surface of plasma membrane. Substantial line-broadening of the NMR resonances of ${ }^{15} \mathrm{~N}$-labeled talin-F2F3 occurred upon adding POPS or PIP2 LUV, whereas much less change was detected when the neutral POPC was added (Supplementary information, Figure S2A). The line-broadening effects in talin-F2F3 induced by all three lipids was concentration-dependent with PIP2 exerting the most 

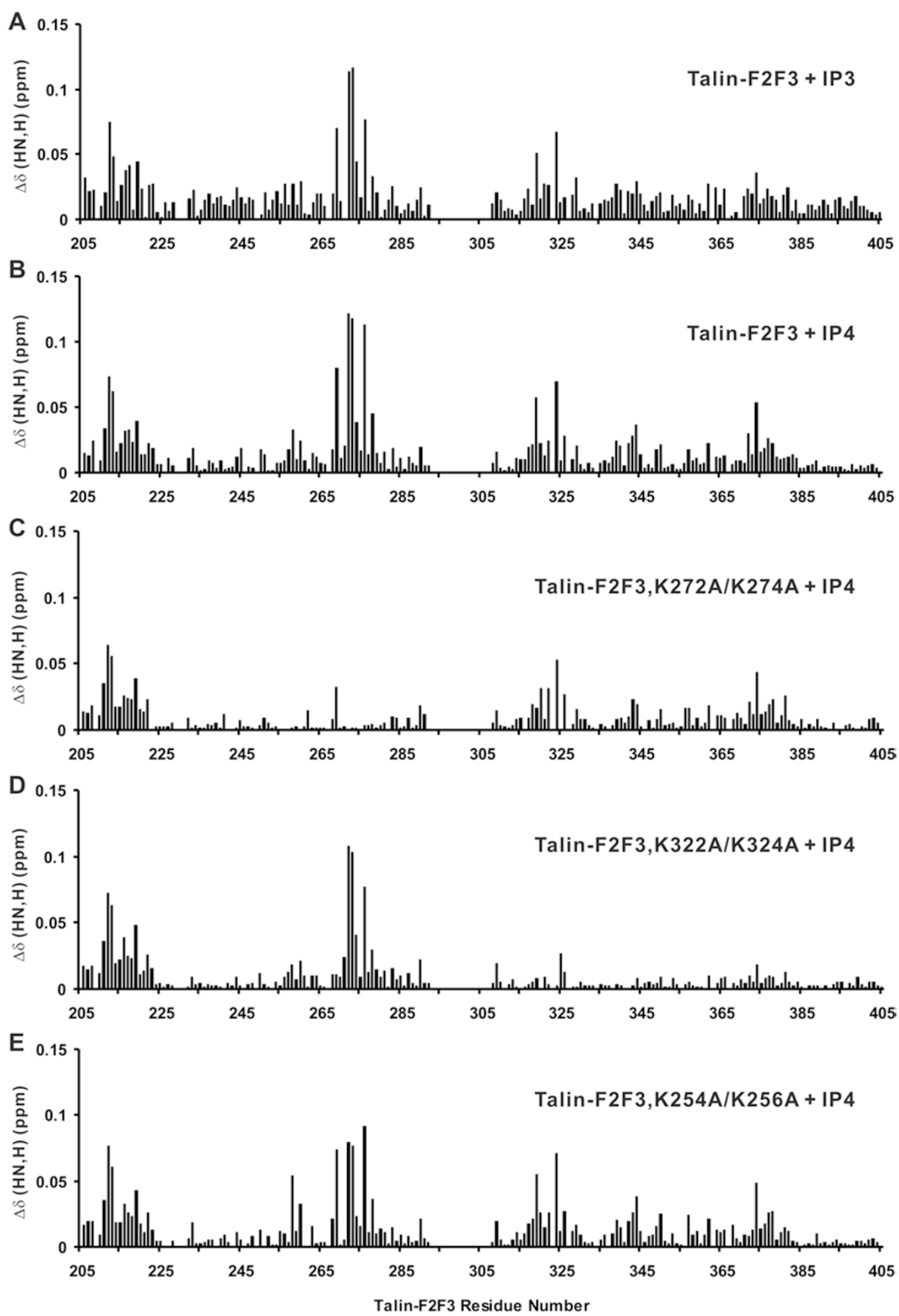

Figure 3 Mapping of PIP2 and PIP3-binding sites on talin-F2F3 using the corresponding head groups IP3 and IP4, respectively. ${ }^{1} \mathrm{H} /{ }^{15} \mathrm{~N}$ chemical shift changes of $0.04 \mathrm{mM}$ talin-F2F3 in the presence of (A) $0.8 \mathrm{mM}$ IP3 or (B) $0.2 \mathrm{mM}$ IP4. Both ligands reveal two major similarly perturbed regions with IP4 having a stronger effect likely due to additional negatively charged phosphate group. (C-E) Perturbation profiles of $0.04 \mathrm{mM} \mathrm{K272A/K274A} \mathrm{(C),} 0.04 \mathrm{mM} \mathrm{K322A/K324A} \mathrm{(D),} \mathrm{and} 0.04 \mathrm{mM} \mathrm{K254A/}$ $\mathrm{K} 256 \mathrm{~A}(\mathrm{E})$ talin-F2F3 in the presence of $0.2 \mathrm{mM}$ IP4. We note that the N-terminal region of talin-F2F3 is also somewhat perturbed, which is insensitive to these mutations and thus the perturbation is probably due to some secondary effect. 
dramatic effect, that is, showing the steepest signal intensity drop at the same protein-lipid ratio (Supplementary information, Figure S2B). Thus, PIP2-containing LUV exhibit the strongest binding to talin-F2F3, which is consistent with the higher negative charge on its head group than POPS and POPC.

Next, we asked structurally how PIP2 recognizes talin-F2F3. NMR-based HSQC spectra were collected for ${ }^{15} \mathrm{~N}$-labeled talin-F2F3 in the absence and presence of increasing concentrations of inositol-1,4,5-trisphosphate (IP3), the head group of PIP2, to obtain molar ratios of $1: 0.0,1: 5.0$, and 1:20.0. To examine the effect of negative charges, we also included inositol-1,3,4,5-tetraphosphate (IP4), the head group of $\mathrm{PI}(3,4,5) \mathrm{P} 3$, at the same molar ratios. Figure 3A and 3B show the detailed chemical shift perturbation profiles of ${ }^{15} \mathrm{~N}$-labeled talin-F2F3 obtained with IP3 and IP4, respectively. Both profiles revealed a major perturbation region (K268 K278) in F2 that is rich in positively charged amino acids, consistent with previous functional analysis [15]. Another positively charged region (K318 K324) in F3 is also perturbed, but less extensively. The effect of IP4 was more pronounced than that with IP3, presumably due to the additional negatively charged phosphate group. Both IP3 and IP4 are quite selective, because, under the same conditions, neither perturbed talin-F0F1 (Supplementary information, Figure S3A), which also has positively charged surface for potential interaction with membranes [16]. This specificity is consistent with the talinF2F3 being the major fragment for the lipid binding [14]. To determine the lipid-binding sites on talin-F2F3 more precisely, we examined the IP4 binding of various talinF2F3 mutants with mutations at sites that are positively charged and perturbed by lipid head groups, including K272A-K274A (on F2) and K322A-K324A (on F3). As a control to examine specificity, we also mutated a positively charged site (K254A-K256A on F2) that is not significantly perturbed. Interestingly, compared to wildtype talin-F2F3, K272A-K274A and K322A-K324A exhibited markedly reduced perturbation at the mutated sites, but little change at the non-mutated sites, indicating that these two sites are independent, each binding to a lipid molecule (Figure 3C and 3D). This interpretation is supported by the isothermal titration calorimetry (ITC) experiment, where talin-H was found to bind PIP2 at a 1:2 ratio (Supplementary information, Figure S3B) and NMR data showing that IP3 binds to these talinF2F3 mutants in similar patterns as IP4 (Supplementary information, Figure S3C and S3D). As a comparison, the control mutant, K254A-K256A, showed comparable binding to IP4 (Figure 3E) or IP3 (Supplementary information, Figure S3E) as wild type with similar chemical shift perturbation profiles. This indicates that the K254K256 site is not geometrically optimized for interacting with the lipid head group despite being positively charged. The bivalent binding mode of PIP2 to talinF2F3 is consistent with the talin-F2F3 structure where (i) the closest distance between the two sites is at least $23 \AA$ that is much larger than a single PIP2 binding pocket (4$11 \AA$ ); and (ii) both sites point towards the membrane, allowing the binding of two PIP2 (Figure 1D, right panel). Since talin-F2 is perturbed more substantially than talin-F3 by PIP2 (Figure 3A), it is possible that the former binds more tightly to the lipids than the latter, which is consistent with the ITC-based affinity estimation for PIP2 with $\mathrm{KD}_{1}$ at $\sim 0.4 \mu \mathrm{M}$ and $\mathrm{KD}_{2}$ at $\sim 5 \mu \mathrm{M}$, respectively (Supplementary information, Figure S3B). The different affinities suggest that the two PIP2 molecules bind talin in a progressive manner in which talinF2 with the higher affinity first anchors to PIP2 and then promotes the second PIP2 binding to talin-F3.

\section{Membrane enriched with PIP2 activates talin via a pull- push mechanism}

With the above data showing that PIP2 binds bivalently to talin-F2F3, we next set out to determine how such binding induces talin activation. As mentioned above, the existing hypothesis is that membrane containing PIP2 might sterically induce a conformational change of talin by binding to talin- $\mathrm{H}[2,15,16,19-21]$, a scheme that is typically observed in other PIP2-binding proteins, including FERM proteins such as moesin [29, $30]$. However, the bipolar membrane-facing surface in our autoinhibited talin structure with the exposed PIP2binding epitope (Figure 1D) suggests a rather different mechanism, which involves a "pull-push" process to relieve the dual inhibition of talin. In this process, the locally enriched PIP2 strongly "pulls" the positively charged surfaces of talin-F2 and F3 to the membrane surface and simultaneously "pushes" down the negatively charged surface of talin-RS (Figure 4A). The opposite movement of the two subunits would clearly cause the mismatch of the interface and the subsequent disruption of the talin-F3/talin-RS complex (Figure 4A). To test this hypothesis, we performed multiple independent experiments. First, we performed NMR-based competition experiment. Comparison of signals obtained for the free talin-RS (black) with those of the talin-F2F3-bound form (blue) shows that a significant number of signals were changed or broadened upon addition of talin-F2F3 (Figure 4B and Supplementary information, Figure S4A), indicative of the interaction between the two fragments. However, when PIP2-enriched LUV were introduced to this complex, essentially all the perturbed talin- 
A

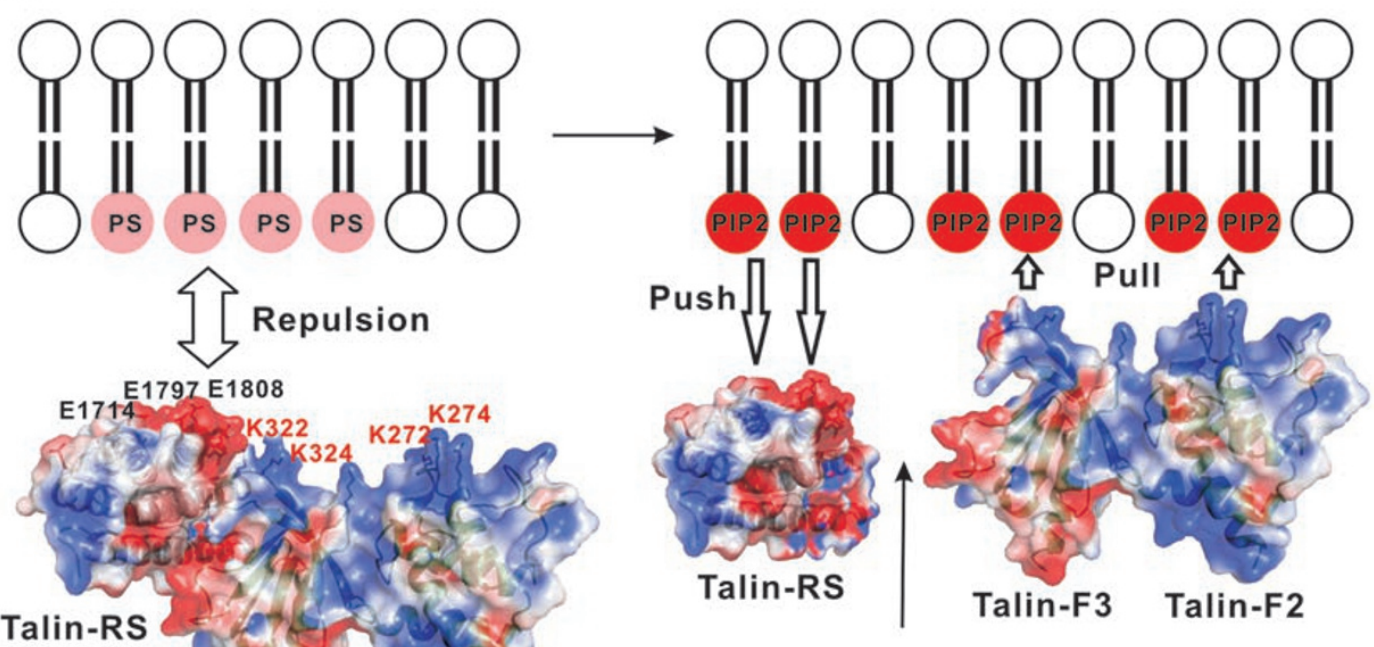

Interface Mismatch

Talin-F3 Talin-F2

B

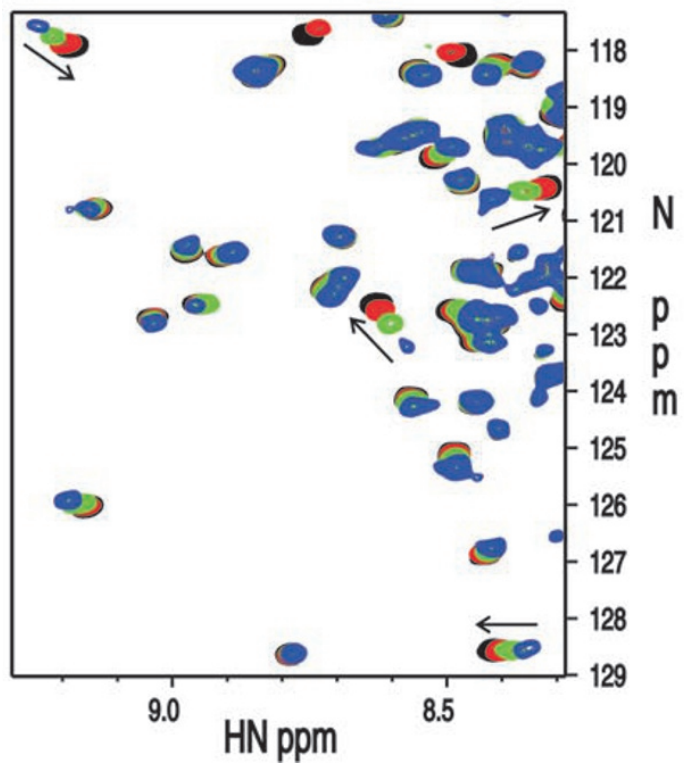

C

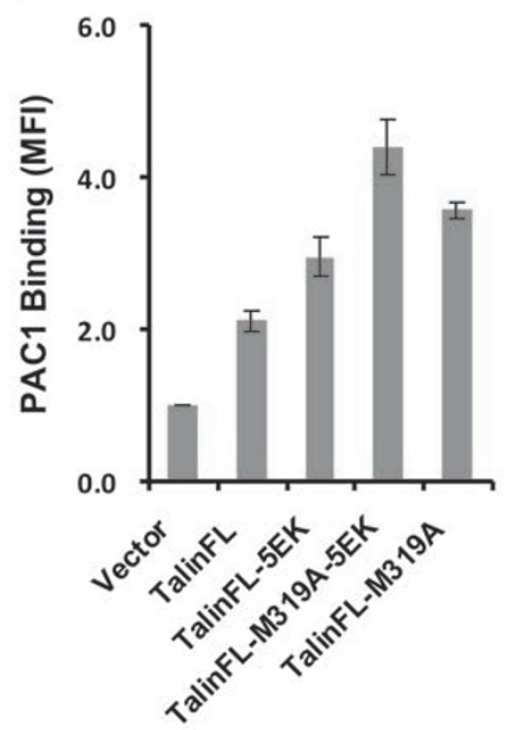

Figure 4 Membrane enriched with PIP2 favorably interacts with talin-F2F3 and promotes talin unmasking via a pull-push mechanism. (A) A structural view to show how unstimulated membrane surface would repel the latent talin (left panel), whereas PIP2-enriched membrane lipids strongly "pulls" up the talin-F2F3 and "pushes" down talin-RS, leading to the interface mismatch and unmasking of the integrin site on talin-FERM (right panel). The blue area indicates region enriched with positive charge and red area indicates region enriched with negative charge. (B) Representative HSQC region of $0.06 \mathrm{mM}$ ${ }^{13} \mathrm{C}-{ }^{15} \mathrm{~N}$-labeled talin-RS in the absence (black) and presence of $0.05 \mathrm{mM}$ talin-F2F3 (blue) or $0.05 \mathrm{mM}$ talin-F2F3 and $3 \mathrm{mM}$ 4:1 POPC:PIP2 LUV (red) or 0.05 mM talin-F2F3 K272A/K274A mutant and 3 mM 4:1 POPC:PIP2 LUV (green). The arrows show that the addition of PIP2 vesicle competes effectively with talin-RS to talin-F2F3, but much less effectively with the talinF2F3 K272A/K274A mutant. (C) Comparison of the activation of integrin $\alpha$ llb $\beta 3$ by vector control, full-length talin (Talin), fulllength talin E1714K/E1794K/E1797K/E1798K/E1808K (Talin 5EK), full-length talin M319A+5EK (Talin M319A-5EK), and fulllength talin M319A (Talin M319A). Both Talin 5EK and Talin M319A are more active than Talin through different mechanisms (see the text). As expected, the combination of M319A and 5EK is more active than the individual ones $(P<0.007$ for Talin vs Talin 5EK, $P<0.001$ for Talin vs Talin M319, and $P<0.002$ for Talin 5EK vs Talin M319A-5EK). Note that WT talin has some basal activity as shown before [19], consistent with that there is an equilibrium between inactive and active talin. The data are representative of seven experiments. 
RS signals either reappeared or returned towards the positions in free form (red spectrum in Figure $4 \mathrm{~B}$ and Supplementary information, Figure S4A), thus providing evidence that PIP2 "pulled" talin-F2F3 to membrane surface, and simultaneously "pushes" away talin-RS to induce the mismatch and disruption of the talin-F3/talinRS interface. By contrast, LUV containing the same percentage of POPS as PIP2 failed to exert the "pull" effect (Supplementary information, Figure S4B) due to its weaker binding to talin-F2F3 than PIP2. Second, to further evaluate this pull-push process, we repeated the experiments with the $\mathrm{K} 272 \mathrm{~A} / \mathrm{K} 274 \mathrm{~A}$ mutant that loses the PIP2 binding in talin-F2, but retains the PIP2 binding capacity in talin-F3 and the talin-RS/talin-F2F3 interaction (Supplementary information, Figure S4C; note that the mutation site is far away from the talinRS/talin-F3 autoinhibitory interface, see Figure 4A). As shown in Figure 4B, the same PIP2 LUV had a substantially reduced capacity to disrupt the talin-RS/talinF2F3 mutant interaction (green), which is consistent with the weakened "pull" mechanism. Finally, to gain cellular functional evidence for the pull-push mechanism, we made a cluster of five charge-reversal mutations on talin-RS (E1714K/E1794K/E1797K/E1798K/ E1808K, 5EtoK; see Figure 1D, left panel) alone and in combination with the constitutively active talin mutant M319A [19] (M319A/5EtoK). The charge-reversal mutation sites are outside the autoinhibitory interface, but the mutations introduced would significantly reduce or reverse the negative charge on talin-RS, thereby facilitating the talin recruitment to membrane (Supplementary information, Figure S5, left panel) - a process that is inhibited for the WT talin (Figure 4A, left panel). Furthermore, upon the recruitment of talin-RS mutant (5EtoK) to the membrane surface, the positively charged surface of talin-F2F3 would further move upward by $\sim 7$ $\AA$ to touch the membrane, because the membrane-facing plane of talin-F2F3 lags behind that of talin-RS based on our structure (Supplementary information, Figure S5). Such upward movement would also lead to the interface mismatch (Supplementary information, Figure S5, right panel) in a way that mimics the intermediate step of the PIP2-mediated pull-push process in which talin-F2F3 moves upward to the membrane while talin-RS is being pushed down (Figure 4A, right panel). As expected, Figure $4 \mathrm{C}$ shows that the 5 EtoK mutant is more active than WT talin, but less active than the M319A/5EtoK mutant. The stronger effect of M319A/5EtoK is clearly due to the additional effect of M319A that disrupts the autoinhibitory interface (Figure 1C and Supplementary information, Figure S1B). Thus, we have obtained combined structural, biochemical, and functional evidence to strongly support the membrane-dependent mechanism of talin activation.

\section{Discussion}

Integrin activation and its coupling to actin are vital to almost all cell-ECM-dependent physiological and pathological processes, such as blood coagulation, tissue remodeling, and cancer metastasis [10-12]. The importance of talin in this dynamic process is undisputed based on the large amount of literatures over the decades $[5,10-12]$, but how talin is spatiotemporally turned on and off has remained elusive despite past significant efforts [2]. The crystal structure of the talin-F2F3/talinRS complex now provides the first definitive view of the "off" state of talin, showing how the integrin binding to talin-FERM is sterically blocked by talin-R (Figure 1B). Moreover, the structure also reveals a previously unrecognized charge-charge repulsion mechanism via talin-RS that electrostatically inhibits the talin-FERM binding to membrane (Figure 4A, left panel). Since the membrane-binding of talin-FERM is essential for its access to integrin $\beta$ CT membrane-proximal region and for enhancing the talin-FERM/integrin interaction [1317], the repulsion mechanism by talin-RS may represent an effective way to negatively regulate this targeting process, which in turn promotes the cytosolic retention of the latent talin. A recent report suggested that talin- $\mathrm{R}$ 482-787 fragment (talin-R1) may also interact with talin-F2F3 and inhibits the talin-FERM/membrane interaction [32]. However, as compared to the potent talin-RS/ talin-F2F3 interaction, the binding of talin-R1 to talinF2F3 appears to be very weak or non-specific, since no significant chemical shift changes were observed even when talin-R1 was in excess $[19,32]$ except for some NMR line-broadening that occurred when even more excess talin-R1 was added to ${ }^{15} \mathrm{~N}$-labeled talin-F2F3 [32]. Further investigation such as obtaining the structures of full-length talin and talin-binding partners is clearly necessary to examine the existence of additional mechanisms that stabilize retention of the latent talin in the cytosol. Nevertheless, our results here have unraveled a distinct regulatory process where the binding of talinFERM to membrane and integrin are dually inhibited by talin-RS. Such dual inhibition likely allows for a tight control of talin activity in physiological settings to minimize spontaneous integrin activation that otherwise could result in severe pathological states, such as thrombosis and tumor progression.

Another significant finding of our study is the mechanism of talin activation by PIP2. Compared to other phosphoinositides that may also bind to talin $[14,21]$, 


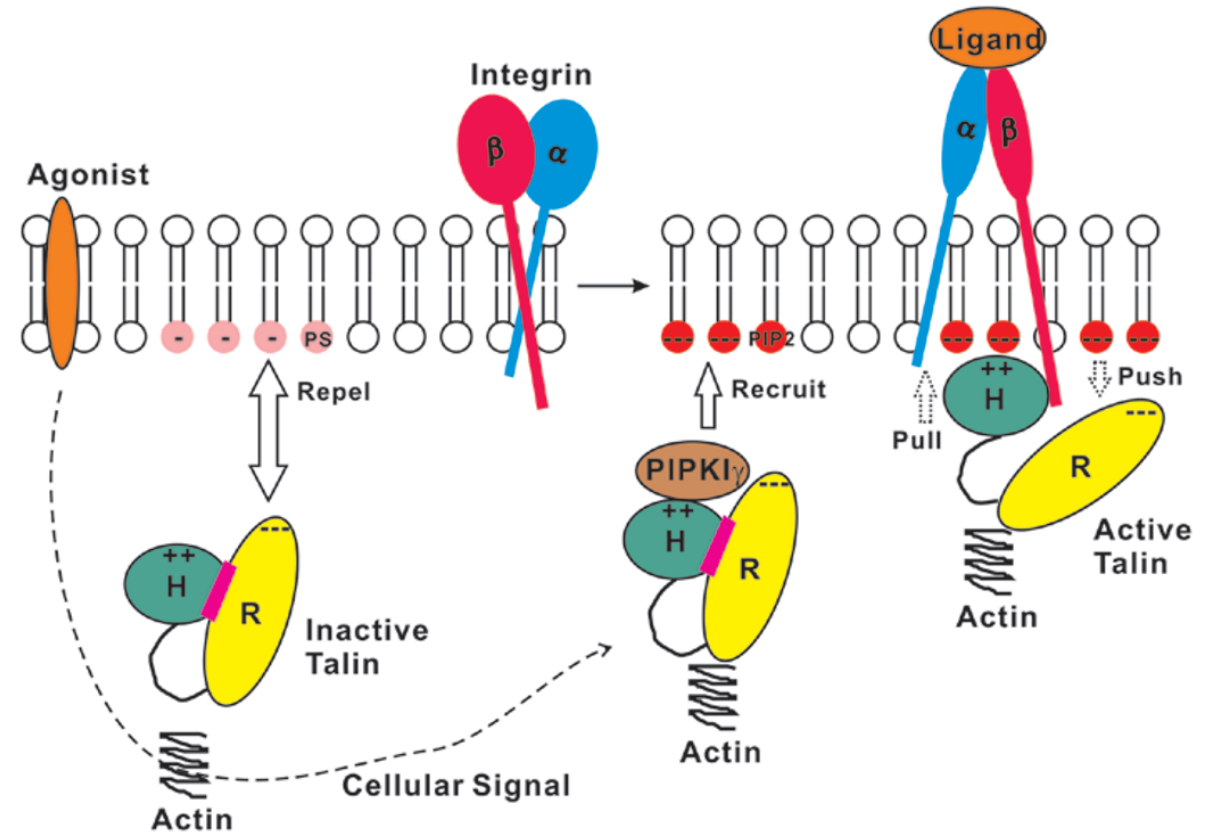

Figure 5 A mechanism of spatiotemporal regulation of talin in mediating integrin activation. In unstimulated cells, talin is autoinhibited and hindered from accessing membrane via the charge-charge repulsion mechanism. Agonist stimulation promotes the co-localization of PIPKI $\gamma$ with talin, thereby leading to the local enrichment of PIP2 for activating talin via the electrostatic pull-push mechanism. The electrostatic pull-push process is different from known steric occlusion by PIP2 that directly interferes with the autoinhibitory protein interfaces [34, 35].

PIP2 is locally enriched by talin-recruited PIPKI $\gamma$ kinase in vivo $[22,23]$ and is thus a physiologically important ligand for talin to promote integrin adhesion $[15,21,25]$. This selectivity is supported by the recent genetic studies where specific ablation of PIPKI $\gamma$ in focal adhesions significantly impaired the talin recruitment to membrane and adhesion sites [28]. Using NMR, we have defined here a bivalent binding mode of PIP2 to talin F2F3, which is different from the classical phosphoinositide binding mode to other known modules [33]. The bivalent PIP2 sites on talin-F2 and F3 are also distinct from the previously reported monovalent PIP2 site in radixin that is located in a groove between F1 and F3 [34], but would promote strong interactions of the membrane with talin-FERM. Thus, PIP2-binding modes to FERM domain are versatile. We then showed that the PIP2-enriched membrane attracts talin-F2F3 and simultaneously repels talin-RS, leading to the relief of the dual inhibition of talin-FERM via an electrostatic pull-push mechanism. This pull-push process differs from the previously proposed steric-clash mechanism $[2,15,16,19,20]$, and the latter is well-known for conventional PIP2-induced activation of proteins including FERM domain proteins moesin [34] and radixin [35], where the PIP2-binding site is buried and can be unmasked by PIP2 via steric occlusion $[29,30]$. Based on these observations, we envision a cascade of molecular events for the membranemediated spatiotemporal regulation of talin-FERM inhibition and activation (Figure 5): (a) In the unstimulated cells, inactive talin is maintained in the cytosol where its FERM domain is unable to bind membrane and integrin. (b) Upon cellular stimulation, PIPKI $\gamma$ is recruited by talin, and promotes local PIP2 enrichment. Note that based on enzyme chemistry, only a tiny amount of PIPKI $\gamma$ is necessary to produce large quantity of PIP2 locally once it associates with some fraction of the highly abundant talin in cells. (c) The membrane with enriched PIP2 recruits talin by strongly "pulling" talin-FERM to the membrane surface and "pushing" away talin-R. This then results in the unmasking of the integrin-binding site on talin-FERM and subsequent talin/integrin interaction and integrin activation. The stronger affinity of PIP3 for talin-F2F3 suggests the involvement of PI3K, which may convert PIP2 into PIP3 to activate talin more potently. This is consistent with the recent finding that knockdown of PIPKI $\gamma$ reduced PIP2 levels by $40 \%$ and PIP3 to undetectable levels [36]. PI3K is known to promote integrin activation [37], but detailed elucidation of this process will require future investigations in vivo. We note that in our competition experiment (Figure 
4B), PIP2 did not completely convert inactive talin into the active state, suggesting an equilibrium between the two states that are balanced by the PIP2 composition of the membrane and possibly other factors. For example, RAP1-RIAM has been suggested to promote the membrane recruitment of talin and regulate integrin activation [38]. Although the exact mechanism remains to be determined, it is possible that RAP1-RIAM cooperates with PIP2-containing membrane in a combinatorial fashion to enhance the equilibrium shift from inactive state to active state of talin.

In summary, we have defined a novel on/off switch mechanism of talin-FERM in controlling integrin transmembrane signaling. In particular, our results unravel a unique role of membrane surface in this on/off switch process, i.e., membrane may act as a dynamic sensor, depending upon its surface chemical environment, to delocalize/inhibit or recruit/activate talin so as to rapidly and effectively communicate with integrins to mediate the integrin activation. Since talin binds to actin via the C-terminal end of the talin-R, such membrane-dependent talin/integrin interaction may also provide means for regulating the mechanical coupling of the integrinactin linkage as well as membrane-actin linkage (Figure $5)$, thereby promoting cytoskeleton reorganization with a profound effect on diverse cellular events, such as cell migration, cell spreading, cell proliferation, and survival.

\section{Materials and Methods}

\section{Peptide and protein preparation and purification}

Talin fragment talin-F0F1 (1-206) was subcloned into the parallel His-1 vector containing a TEV-cleavable N-terminal His tag. The DNA sequence was verified and transformed into $E$. coli. The protein was purified according to the native purification protocol from Qiagen. The His tag was removed and the protein was further purified using size-exclusion chromatography on Superdex 75 (Amersham Bioscience). Other talin fragments including talinF3 (309-405) with a solubility improving mutation C336S, talinF2F3 (206-405), talin-RS (1654-1848), and remaining talin-R fragments were prepared as described previously [19]. ${ }^{15} \mathrm{~N}$ and/ or ${ }^{13} \mathrm{C}$ isotope labeling was achieved by employing ${ }^{15} \mathrm{NH}_{4} \mathrm{Cl}(1.1$ $\mathrm{g} / \mathrm{l})$ and/or ${ }^{13} \mathrm{C}$ glucose $(3 \mathrm{~g} / \mathrm{l})$ as the sole nitrogen and carbon sources in the cultures. ${ }^{2} \mathrm{H}$ isotope labeling was achieved by using ${ }^{2} \mathrm{H}$ glucose $(3 \mathrm{~g} / \mathrm{l})$ and preparing the culture in $99.8 \% \mathrm{D}_{2} \mathrm{O}$. Double mutation of talin-F2F3 (K254A/K256A, K272A/K274A, and K322A/K324A) were made using QuickChange Site-Directed Mutagenesis (Stratagene) and prepared in the same way as wild type.

\section{Lipid vesicle preparation}

POPC, POPS, and PIP2 were purchased from Avanti Polar Lipids (Alabaster, AL, USA). LUVs of either single or mixed lipid were prepared by extrusion. Briefly, lipids were first dissolved together in chloroform. The chloroform was then removed under a stream of nitrogen followed by overnight vacuum pumping. The lipid film was hydrated in desired buffer, followed by homogenization with a few freeze-thaw cycles. The LUV was finally formed by extruding the lipid suspension $\sim 20$ times through two stacked $0.1 \mu \mathrm{m}$ polycarbonate filters (Avanti Polar Lipids).

\section{Crystallization, data collection, and structure determina- tion}

Crystals of the talin-F2F3/talin-RS complex were grown at $16{ }^{\circ} \mathrm{C}$ by the hanging-drop vapor diffusion method in drops containing a 2:1 $(\mathrm{v} / \mathrm{v})$ ratio of protein solution $(\sim 0.2 \mathrm{mM})$ and reservoir solution consisting of $100 \mathrm{mM}$ MES, pH 6.5, 18.5\% (w/v) polyethylene glycol 1500. Data sets were collected at $100 \mathrm{~K}$ at the beamline BL17U at the Shanghai Synchrotron Radiation Facility (Shanghai, China) using an ADSC Q315 CCD detector. All X-ray intensity data were integrated and scaled using the programs HKL2000 [39]. The structure was determined by the molecular replacement method with a crystal structure of talin-F2F3 (PDB entry: 3G9W) and an NMR structure of talin-RS (PDB entry: 2KBB) as starting models. Manual model building was carried out by the program COOT [40] and completed by TURBO-FRODO [41]. The model was refined by iterative rounds of manual rebuilding followed by PHENIX [42] and Refmac5 [43]. Stereochemistry of the structures was assessed by PROCHECK [44]. Shape correlation statistics were calculated by SC [45]. Structures are visualized with PyMOL (Schrodinger, LLC. http://www.pymol. org), and the surface electrostatic potential was calculated with the program APBS [46].

\section{NMR spectroscopy}

All heteronuclear NMR experiments were performed at $25{ }^{\circ} \mathrm{C}$ on Bruker Avance $600 \mathrm{MHz}$ and $900 \mathrm{MHz}$ spectrometers, equipped with cryogenic triple resonance probes and shielded $z$-gradient units. All the NMR samples contain $5 \%(\mathrm{v} / \mathrm{v})$ of ${ }^{2} \mathrm{H}_{2} \mathrm{O}$ and $1 \mathrm{mM}$ DSS. Titrations of wild-type and double-mutated talin-F2F3 with various ratios of different LUVs or IP3/IP4 were conducted with $0.04 \mathrm{mM}{ }^{15} \mathrm{~N}$-labeled protein in MES buffer (20 mM MES, 20 $\mathrm{mM} \mathrm{NaCl}, 2 \mathrm{mM} \mathrm{NaN}_{3}$, and $\mathrm{pH}$ 6.1). The HSQC experiments to examine the autoinhibitory talin complex were performed with 0.06 $\mathrm{mM}{ }^{13} \mathrm{C} /{ }^{15} \mathrm{~N}$-labeled talin-RS and $0.05 \mathrm{mM}$-unlabeled talin-F2F3 in phosphate buffer $\left(50 \mathrm{mM} \mathrm{Na} \mathrm{HPO}_{4} / \mathrm{NaH}_{2} \mathrm{PO}_{4}, 50 \mathrm{mM} \mathrm{NaCl}\right.$, $2 \mathrm{mM} \mathrm{NaN}_{3}$, and $\mathrm{pH}$ 6.8). The effects of membrane lipids were examined with the addition of $3 \mathrm{mM}$ desired LUV to the complex. The weighted chemical shift changes of amide proton and nitrogen were calculated using the equation: $\Delta \delta_{\mathrm{obs}[\mathrm{HN}, \mathrm{N}]}=\left(\left[\Delta \delta_{\mathrm{HN}} \mathrm{W}_{\mathrm{HN}}\right]^{2}+[\Delta \delta\right.$ $\left.\left.{ }_{\mathrm{N}} \mathrm{W}_{\mathrm{N}}\right]^{2}\right)^{1 / 2}$, where $\mathrm{W}_{\mathrm{HN}}(1.0)$ and $\mathrm{W}_{\mathrm{N}}(0.154)$ are weighting factors based on the gyromagnetic ratios of ${ }^{1} \mathrm{H}$ and ${ }^{15} \mathrm{~N}$. NMR data were processed and analyzed using nmrPipe [47], PIPP [48], and Sparky [49].

\section{Isothermal calorimetry measurements}

Isothermal titration calorimetry was performed using a VPITC instrument (MicroCal). Talin-H and talin-F2F3 were dialyzed against a buffer containing $100 \mathrm{mM} \mathrm{NaCl}$ and $50 \mathrm{mM} \mathrm{Na}_{2} \mathrm{HPO}_{4} /$ $\mathrm{NaH}_{2} \mathrm{PO}_{4}$ at $\mathrm{pH}$ 6.8. D-myo-phosphatidylinositol 4,5-bisphoshate or PIP2-diC8 (Echelon Biosciences) was dissolved in the same buffer to a final concentration of $1.2 \mathrm{mM}$. Titrations consisted of 30 injections of $7.5 \mu 1$ PIP2-diC8 stock into the sample cell containing $50 \mu \mathrm{M}$ of $1.4 \mathrm{ml}$ Talin-H protein. A control experiment was performed to estimate the heat of dilution and heat of 
mixing by injecting the ligand after saturation of the experiment. This was subtracted from all data points prior to curve fitting. The data were fitted into a two site binding model and thermodynamic parameters were calculated using Origin software provided by MicroCal. All experiments were performed at $25^{\circ} \mathrm{C}$.

\section{Plasmids, mutagenesis, and transfections}

The preparation of plasmids for mouse full-length talin 1 and M319A mutant and their transfections were described previously [19]. Other mutations used for this study were performed using QuickChange Site-Directed Mutagenesis Kit (Stratagene).

\section{Integrin activation assay}

The effects of full-length talin, full-length talin mutants, and talin- $\mathrm{H}$ on integrin activation were analyzed using $\mathrm{CHO}$ cells stably expressing integrin ( $\alpha \mathrm{IIb} \beta 3-\mathrm{CHO})$ and an activation-specific anti- $\alpha \operatorname{IIb} \beta 3 \mathrm{mAb}$ (PAC1) as described previously [19].

\section{Acknowledgments}

This work was supported by grants awarded to JQ (NIH GM62823), EFP (NIH P01HL073311), and RZ (2011CB911101). We thank Qin lab members at Lerner Research Instittue, Xiaolun Zhang, Julia Vaynberg, Yan-Qing Ma, Koichi Fukuda, Sujay Ithychanda, and Jianmin Liu for assistance and useful discussions. We also thank Xiaolan Zhao and Satya Yadav in Lerner Research Institute core facilities for technical assistance of our experiments. The atomic coordinates of talin-F2F3/talin-RS complex have been deposited in the Protein Data Bank with the accession code 4F7G.

\section{References}

1 Burridge K, Connell L. A new protein of adhesion plaques and ruffling membranes. $J$ Cell Biol 1983; 97:359-367.

2 Critchley DR. Biochemical and structural properties of the integrin-associated cytoskeletal protein talin. Annu Rev Biophys 2009; 38:235-254.

3 Moser M, Legate KR, Zent R, Fassler R. The tail of integrins, talin, and kindlins. Science 2009; 324:895-899.

4 Wehrle-Haller B. Structure and function of focal adhesions. Curr Opin Cell Biol 2012; 24:116-124.

5 Turner CE, Burridge K. Transmembrane molecular assemblies in cell-extracellular matrix interactions. Curr Opin Cell Biol 1991; 3:849-853.

6 Calderwood DA, Zent R, Grant R, Rees DJ, Hynes RO, Ginsberg MH. The Talin head domain binds to integrin beta subunit CTs and regulates integrin activation. $J$ Biol Chem 1999; 274:28071-28074.

7 Vinogradova O, Velyvis A, Velyviene A, et al. A structural mechanism of integrin alpha(IIb)beta(3) "inside-out" activation as regulated by its cytoplasmic face. Cell 2002; 110:587597.

8 Kim M, Carman CV, Springer TA. Bidirectional transmembrane signaling by cytoplasmic domain separation in integrins. Science 2003; 301:1720-1725.

9 Tadokoro S, Shattil SJ, Eto K, et al. Talin binding to integrin beta tails: a final common step in integrin activation. Science 2003; 302:103-106.

10 Hynes RO. Integrins: bidirectional, allosteric signaling ma- chines. Cell 2002; 110:673-687.

11 Qin J, Vinogradova O, Plow EF. Integrin bidirectional signaling: a molecular view. PLoS Biol 2004; 2:e169.

12 Luo BH, Carman CV, Springer TA. Structural basis of integrin regulation and signaling. Annu Rev Immunol 2007; 25:619-647.

13 Anthis NJ, Wegener KL, Ye F, et al. The structure of an integrin/talin complex reveals the basis of inside-out signal transduction. EMBO J 2009; 28:3623-3632.

14. Moore DT, Nygren P, Jo H, Boesze-Battaglia K, Bennett JS, Degrado WF. Affinity of talin- 1 for the $\beta 3$-integrin cytosolic domain is modulated by its phospholipid bilayer environment. Proc Natl Acad Sci USA 2012; 109:793-798.

15 Saltel F, Mortier E, Hytonen VP, et al. New PI(4,5)P2- and membrane proximal integrin-binding motifs in the talin head control beta3-integrin clustering. J Cell Biol 2009; 187:715731.

16 Elliott PR, Goult BT, Kopp PM, et al. The structure of the talin head reveals a novel extended conformation of the FERM domain. Structure 2010; 18:1289-1299.

17 Kalli AC, Wegener KL, Goult BT, Anthis NJ, Campbell ID, Sansom MS. The structure of the talin/integrin complex at a lipid bilayer: an NMR and MD simulation study. Structure 2010; 18:1280-1288.

18 Cox D, Brennan M, Moran N. Integrins as therapeutic targets: lessons and opportunities. Nat Rev Drug Discov 2010; 9:804-820.

19 Goksoy E, Ma YQ, Wang X, et al. Structural basis for the autoinhibition of talin in regulating integrin activation. Mol Cell 2008; 31:124-133.

20 Goult BT, Bate N, Anthis NJ, et al. The structure of an interdomain complex that regulates talin activity. $\mathrm{J}$ Biol Chem 2009; 284:15097-15106.

21 Martel V, Racaud-Sultan C, Dupe S, et al. Conformation, localization, and integrin binding of talin depend on its interaction with phosphoinositides. J Biol Chem 2001; 276:2121721227.

22 Ling K, Doughman RL, Firestone AJ, Bunce MW, Anderson RA. Type I gamma phosphatidylinositol phosphate kinase targets and regulates focal adhesions. Nature 2002; 420:8993.

23 Di Paolo G, Pellegrini L, Letinic K, et al. Recruitment and regulation of phosphatidylinositol phosphate kinase type 1 gamma by the FERM domain of talin. Nature 2002; 420:8589.

24 Hinchliffe KA, Irvine RF, Divecha N. Aggregation-dependent, integrin-mediated increases in cytoskeletally associated PtdInsP2 $(4,5)$ levels in human platelets are controlled by translocation of PtdIns 4-P 5-kinase $\mathrm{C}$ to the cytoskeleton. EMBO J 1996; 15:6516-6524.

25 Cluzel C, Saltel F, Lussi J, Paulhe F, Imhof BA, WehrleHaller B. The mechanisms and dynamics of (alpha)v(beta)3 integrin clustering in living cells. J Cell Biol 2005; 171:383392.

26 Bolomini-Vittori M, Montresor A, Giagulli C, et al. Regulation of conformer-specific activation of the integrin LFA-1 by a chemokine-triggered Rho signaling module. Nat Immunol 2009; 10:185-194.

27 García-Bernal D, Parmo-Cabañas M, Dios-Esponera A, Sa- 
maniego R, Hernán-P de la Ossa D, Teixidó J. Chemokineinduced Zap70 kinase-mediated dissociation of the Vav1-talin complex activates alpha4betal integrin for $\mathrm{T}$ cell adhesion. Immunity 2009; 31:953-964.

28 Legate KR, Takahashi S, Bonakdar N, et al. Integrin adhesion and force coupling are independently regulated by localized PtdIns(4,5)(2) synthesis. EMBO J 2011; 30:4539-4553.

29 Di Paolo G, De Camilli P. Phosphoinositides in cell regulation and membrane dynamics. Nature 2006; 443:651-657.

30 Groves JT, Kuriyan J. Molecular mechanisms in signal transduction at the membrane. Nat Struct Mol Biol 2010; 17:659665.

31 García-Alvarez B, de Pereda JM, Calderwood DA, et al. Structural determinants of integrin recognition by talin. Mol Cell 2003; 11:49-58.

32 Banno A, Goult BT, Lee H, Bate N, Critchley DR, Ginsberg $\mathrm{MH}$. Subcellular localization of talin is regulated by interdomain interactions. J Biol Chem 2012; 287:13799-13812.

33 Kutateladze TG. Translation of the phosphoinositide code by PI effectors. Nat Chem Biol 2010; 6:507-513.

34 Li Q, Nance MR, Kulikauskas R, et al. Self-masking in an intact ERM-merlin protein: an active role for the central alpha-helical domain. J Mol Biol 2007; 365:1446-1459.

35 Hamada K, Shimizu T, Matsui T, Tsukita S, Hakoshima T. Structural basis of the membrane-targeting and unmasking mechanisms of the radixin FERM domain. EMBO J 2000; 19:4449-4462.

36 Wu Z, Li X, Sunkara M, Spearman H, Morris AJ, Huang C. PIPKI $\gamma$ Regulates focal adhesion dynamics and colon cancer cell invasion. PLoS One 2011; 6:e24775.

37 Jackson SP, Schoenwaelder SM. PI3-kinase p110beta regulation of platelet integrin alpha(IIb)beta3. Curr Top Microbiol Immunol 2010; 346: 203-224.

38 Watanabe N, Bodin L, Pandey M, et al. Mechanisms and consequences of agonist-induced talin recruitment to platelet integrin alphaIIbbeta3. J Cell Biol 2008; 181:1211-1222.
39 Otwinowski Z, Minor W. Processing of X-ray diffraction data collected in oscillation mode. Method Enzymol 1997; 276:307-326.

40 Emsley P, Cowtan K. Coot: model-building tools for molecular graphics. Acta Crystallogr D Biol Crystallogr 2004; 60:2126-2132.

41 Roussel A, Cambillau C. TURBO-FRODO in Silicon Graphics Geometry Partners Directory. Silicon Graphics: Mountain View, CA, 1991.

42 Adams PD, Afonine PV, Bunkoczi G, et al. PHENIX: a comprehensive Python-based system for macromolecular structure solution. Acta Crystallogr D Biol Crystallogr 2010; 66:213-221.

43 Murshudov GN, Vagin AA, Dodson EJ. Refinement of macromolecular structures by the maximum-likelihood method. Acta Crystallogr D Biol Crystallogr 1997; 53:240-255.

44 Laskowski RA, Macarthur MW, Moss DS, Thornton JM. Procheck - a program to check the stereochemical quality of protein structures. J Appl Crystallogr 1993; 26:283-291.

45 Lawrence MC, Colman PM. Shape complementarity at protein/protein interfaces. J Mol Biol 1993; 234:946-950.

46 Unni S, Huang Y, Hanson RM, et al. Web servers and services for electrostatics calculations with APBS and PDB2PQR. J Comput Chem 2011; 32:1488-1491.

47 Delaglio F, Grzesiek S, Vuister GW, Zhu G, Pfeifer J, Bax A. NMRPipe: a multidimensional spectral processing system based on UNIX pipes. J Biomol NMR 1995; 6:277-293.

48 Garrett DS, Powers R, Gronenborn AM, Clore GM. A common-sense approach to peak picking in 2-dimensional, 3-dimensional, and 4-dimensional spectra using automatic computer-analysis of contour diagrams. J Magn Reson 1991; 95:214-220.

49 Lee W, Westler WM, Bahrami A, Eghbalnia HR, Markley JL. PINE-SPARKY: graphical interface for evaluating automated probabilistic peak assignments in protein NMR spectroscopy. Bioinformatics 2009; 25:2085-2087.

(Supplementary information is linked to the online version of the paper on the Cell Research website.) 